metabolic biomarkers, increasing age, Korean ethnicity, higher disease activity and increased serum leptin performed similarly (AUC ROC=0.75).

Conclusions: SLE patients who develop incident MetS exhibit a more inflammatory disease phenotype, with higher corticosteroid exposure in the preceding visit. Increased serum leptin concentration is independently associated with future onset of MetS. These factors can help predict those at increased risk of developing future MetS and may help target patients for more focused cardiovascular disease prevention.

Disclosure of Interest: None declared

DOI: 10.1136/annrheumdis-2018-eular.4158

\section{OP0119 PROGRESSION OF SUBCLINICAL ATHEROSCLEROSIS IN SYSTEMIC LUPUS ERYTHEMATOSUS OF LOW DISEASE ACTIVITY: THREE-YEAR FOLLOW-UP AND COMPARISON TO RHEUMATOID ARTHRITIS}

E. Kravvariti, G. Konstantonis, P.P. Sfikakis, M.G. Tektonidou. First Department of Propaereutic and Internal Medicine/Joint Rheumatology Program, 'Laikon' Hospital, School of Medicine, National and Kapodistrian University of Athens, Athens, Greece

Background: Both systemic lupus erythematosus (SLE) and rheumatoid arthritis (RA) are characterised by accelerated atherosclerosis compared to the general population. Prospective studies have shown that atherosclerosis progression is halted in patients with RA of low disease activity, but it is unclear if maintaining lupus low disease activity state mitigates accelerated atherosclerosis due to SLE. Objectives: To prospectively assess the risk and determinants of atherosclerosis progression in SLE versus RA patients of low disease activity.

Methods: We performed carotid and femoral artery ultrasound to detect atherosclerotic plaques at baseline on 345 participants with SLE, RA, and healthy controls, individually matched for age and gender, after excluding patients with atherosclerotic cardiovascular disease, malignancy and diabetes. After 3 years of follow-up, patients with SLE $(n=89)$ and RA $(n=64)$ maintaining low disease activity ${ }^{1}$ for $>75 \%$ of the follow-up time, and their matched controls $(n=72)$ underwent repeat ultrasound to identify those with atherosclerosis progression, as defined by the development of new plaques compared to baseline. We applied multiple logistic regression models to assess the odds of atherosclerosis progression between SLE, RA, and control participants, and used the stepwise backward elimination algorithm $(p<0.1)$ to examine potential associations with SLE damage index, antiphospholipid antibodies, corticosteroids, hydroxycloroquine, immunosuppressives, and disease duration in patients with SLE, adjusting for use of antiplatelet agents, statins, and traditional cardiovascular risk factors with the European Society of Cardiology's SCORE risk estimation of 10 year fatal cardiovascular disease.

Results: Atherosclerotic plaque progression was detected in $21 \%$ of SLE patients, $17 \%$ of RA patients, and $8 \%$ of controls $(p=0.078)$. After controlling for SCORE, antiplatelet agent use and statins, the rate of atherosclerosis progression compared to healthy controls was significantly higher in SLE $(O R=3.05$, 95\% Confidence Interval $(\mathrm{Cl}) 1.06-8.79, \mathrm{p}=0.039)$, but not in $\mathrm{RA}(\mathrm{OR}=2.11,95 \% \mathrm{Cl}: 0.72$ to $6.23, p=0.176)$. In patients with SLE, longer disease duration at baseline $(\mathrm{OR}=1.11,95 \% \mathrm{Cl}: 1.02$ to $1.21, \mathrm{p}=0.015)$, antiphospholipid antibody positivity (OR=7.04, 95\% Cl: 1.57 to $31.58, p=0.011$ cumulative corticosteroid dose during follow-up $(\mathrm{OR}=1.16,95 \% \mathrm{Cl}$ : 0.99 to $1.35, \mathrm{p}=0.069)$, treatment with antiplatelet agents $(\mathrm{OR}=0.21,95 \% \mathrm{Cl}: 0.05$ to $0.99, \mathrm{p}=0.049)$, and the SCORE prediction $(\mathrm{OR}=1.67,95 \% \mathrm{Cl}: 0.91$ to $3.08, \mathrm{p}=0.099)$ were included in the multivariable model as determinants of atherosclerosis progression. No disease-related determinants were significantly associated with atherosclerosis progression in patients with RA.

Conclusions: Unlike RA, atherosclerosis progression is accelerated in SLE even in the setting of low disease activity. In addition to SCORE, longer disease duration at baseline, antiphospholipid antibody positivity, and higher cumulative corticosteroid dose during follow-up increase the odds of atherosclerosis progression in patients with SLE of low disease activity.

\section{REFERENCE:}

[1] Franklyn K, et al. Definition and initial validation of a Lupus Low Disease Activity State (LLDAS). Annals of the Rheumatic Diseases 2016;75:161521 .

Disclosure of Interest: None declared DOI: 10.1136/annrheumdis-2018-eular.3038

\section{OP0120} INFLUENCE OF EPIDEMIOLOGY AND ETHNICITY ON SYSTEMIC EXPRESSION OF PRIMARY SJÖGREN SYNDROME IN 9974 PATIENTS

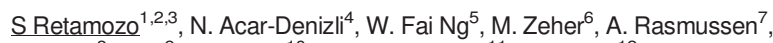
R. Seror ${ }^{8}$, X. Li ${ }^{9}$, C. Baldini ${ }^{10}$, J.-E. Gottenberg ${ }^{11}$, D. Danda ${ }^{12}$, L. Quartuccio ${ }^{13}$, R. Priori ${ }^{14}$, G. Hernandez-Molina ${ }^{15}$, B. Armagan ${ }^{16}$, A.A. Kruize ${ }^{17}$, S.-K. Kwok ${ }^{18}$, M. Wahren-Herlenius ${ }^{19}$, S. Praprotnik ${ }^{20}$, D. Sene ${ }^{21}$, E. Bartoloni ${ }^{22}$, R. Solans ${ }^{23}$,

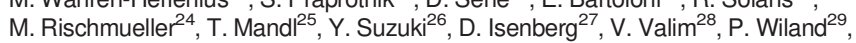
G. Nordmark ${ }^{30}$, G. Fraile ${ }^{31}$, H. Bootsma ${ }^{32}$, T. Nakamura $^{33}$, R. Giacomelli ${ }^{34}$, V. Devauchelle-Pensec ${ }^{35}$, B. Hofauer ${ }^{36}$, M. Bombardieri ${ }^{37}$, V. Fernandes Moça Trevisani $^{38}$, D. Hammenfors ${ }^{39}$, S.G. Pasoto ${ }^{40}$, T.A. Gheita ${ }^{41}$, F. Atzeni ${ }^{42}$ J. Morel ${ }^{43}$, C. Vollenveider ${ }^{44}$, P. Brito-Zerón ${ }^{1,45}$, M. Ramos-Casals ${ }^{1}$, on behalf of the Sjogren Big Data Consortium. ${ }^{1} \mathrm{H}$. Clinic, IDIBAPS, Barcelona, Spain; ${ }^{2} \mathrm{H}$. Privado Cordoba, IUCBC; ${ }^{3}$ NICSA, UNC-CONICET, Cordoba, Argentina; ${ }^{4}$ Mimar Sinan Univ, Istanbul, Turkey; ${ }^{5}$ Newcastle Univ, NHS Foundation Trust, Newcastle, UK; ${ }^{6}$ Debrecen Univ, Debrecen, Hungary; ${ }^{7}$ Oklahoma Medical Research Foundation, Oklahoma, USA; ${ }^{8}$ Université Paris Sud, INSERM, Paris, France; ${ }^{9}$ Anhui Provincial Hosp, Hefei, China; ${ }^{10}$ Pisa Univ, Pisa, Italy; ${ }^{11}$ Strasbourg Univ, CNRS, Strasbourg, France; ${ }^{12}$ Christian Medical College and Hosp, Vellore, India; ${ }^{13}$ Hosp 'Santa Maria della Misericordia', Udine; ${ }^{14}$ Sapienza Univ, Rome, Italy, ${ }^{15}$ INCMNSS, México City, Mexico; ${ }^{16}$ Hacettepe Univ, Ankara, Turkey, ${ }^{17}$ Univ Medical Center, Utrecht, Netherlands; ${ }^{18}$ Catholic Univ of Korea, Seoul, Korea, Republic Of, ${ }^{19}$ Karolinska Institute, Stockholm, Sweden; ${ }^{20}$ Univ Medical Centre, Ljubljana, Slovenia; ${ }^{21}$ Univ Paris VII Publique-Hôpitaux de Paris, 2, Paris, France; ${ }^{22}$ Perugia Univ, Perugia, Italy, ${ }^{23} \mathrm{H}$. Vall d'Hebron, Barcelona, Spain; ${ }^{24}$ Western Australia Univ, Crawley, Australia; ${ }^{25}$ Malmö Hosp, Lund Univ, Lund, Sweden; ${ }^{26}$ Kanazawa Univ Hosp, Ishikawa, Japan; ${ }^{27}$ University College, London, UK; ${ }^{28}$ Federal Univ Espírito Santo, Vitória, Brazil;, ${ }^{29}$ Wroclaw Medical Hospital, Wroclaw, Poland; ${ }^{30}$ Uppsala Univ, Uppsala, Sweden; ${ }^{31} \mathrm{H}$. Ramón y Cajal, Madrid, Spain; ${ }^{32}$ Univ Medical Center, Groningen, Netherlands; ${ }^{33}$ Nagasaki University, Nagasaki, Japan; ${ }^{34}$ L'Aquila Univ, L'Aquila, Italy; ${ }^{35}$ Brest Univ Hosp, CERAINO, Brest, France; ${ }^{36}$ Technische Univ, Munchen, Germany, ${ }^{37}$ Queen Mary Univ, London, USA; ${ }^{38}$ Federal Univ São Paulo, Sao Paulo, Brazil; ${ }^{39}$ Haukeland Univ Hosp, Bergen, Norway, ${ }^{40}$ Hospital das Clínicas, USP, São Paulo, Brazil; ${ }^{41}$ Cairo University, Cairo, Egypt, ${ }^{42}$ Messina and Milan Univ, Milan, Italy; ${ }^{43} \mathrm{Hosp}$ and Univ of Montpellier, Montpellier, France; ${ }^{44}$ German Hosp, Buenos Aires, Argentina; ${ }^{45} \mathrm{H}$. CIMA- Sanitas, Barcelona, Spain

Objectives: To analyse the influence of epidemiology and ethnicity on the clinical systemic presentation at diagnosis of primary Sjögren syndrome (SjS).

Methods: The Big Data Sjögren Database included 10475 worldwide patients from 22 countries fulfilling the 2002 criteria. Age at diagnosis, gender and ethnicity (77\% White, $14 \%$ Asian, 6\% Hispanic, 1\% Black/African American, $2 \%$ others) were correlated with systemic involvement at diagnosis (retrospectively scored in 9974 patients using ESSDAI/ClinESSDAl)

Results: Men had higher mean ESSDAl (8.0 vs $5.9, p<0.001)$ and clinESSDA (8.4 vs $6.1, p<0.001$ ) in comparison with women; the domains more active in men included lymphadenopathy $(p<0.001)$, glandular $(p<0.001)$, pulmonary $(p=0.001)$, PNS $(p<0.001)$ and CNS $(p<0.001)$. Highest scores were also reported in patients with young-onset disease ( $<35$ years) with respect to older onset ( $>65$ years) for ESSDAl (6.5 vs $5.6, p=0.002$ ) and clinESSDAl (6.4 vs $5.8, p=0.034$ ). Highest ESSDAI scores were reported in Black/African American, followed by White, Asian and Hispanic patients (6.7 vs 6.3 vs 5.4 vs 4.9 , respectively, $p<0.001$ ). BAA patients showed the highest frequency of activity in lymphadenopathy, articular PNS, CNS and biological domains, Whites in glandular, cutaneous and muscular, Asians in pulmonary, renal and haematological and Hispanics in the constitutional domain.

Conclusions: This study provides the first evidence for a strong influence of epidemiology and ethnicity on the systemic phenotype at diagnosis of primary SjS

Disclosure of Interest: None declared

DOI: 10.1136/annrheumdis-2018-eular.6073

\begin{tabular}{l|l}
\hline OP0121 & LUPUS ANTICOAGULANT IS ASSOCIATED WITH \\
& THROMBOTIC EVENTS IN HEALTHY CARRIERS: \\
& RESULTS FROM A PROSPECTIVE LONGITUDINAL \\
& STUDY
\end{tabular}

F. Ceccarelli ${ }^{1}$, A. Chistolini ${ }^{2}$, C. Perricone ${ }^{1}$, E. Rando ${ }^{1}$, C. Pirone ${ }^{1}$, E. Cipriano ${ }^{1}$ I. Leccese ${ }^{1}$, L. Massaro ${ }^{1}$, F. Morello ${ }^{1}$, F.R. Spinelli ${ }^{1}$, C. Alessandri ${ }^{1}$, G. Valesini ${ }^{1}$, F. Conti ${ }^{1}{ }^{1}$ Lupus Clinic, Reumatologia; ${ }^{2}$ Dipartimento di Biotecnologie cellulari ed Ematologia, Sapienza Università di Roma, Sapienza University of Rome, Rome, Italy

Background: Antiphospholipid syndrome (APS) is an autoimmune disease characterised by thrombotic events and/or pregnancy morbidity in combination with persistent positivity for antiphospholipid antibodies (aPL) [lupus anticoagulant (LA), anticardiolipin (aCL), anti b2 glycoprotein I (anti-ß2GPI) in medium/high titers]. These antibodies could be identified not only in other pathological 
conditions (i.e. autoimmune diseases), but also in healthy subjects (aPL carriers). In this subset, the risk of developing thrombotic events or pregnancy morbidity ranges from $0 \%$ to $3.8 \%$, and it is higher in subjects with LA positivity, triple positivity $(\mathrm{LA}+\mathrm{aCL}+\mathrm{anti}-\beta 2 \mathrm{GPI})$ and $\mathrm{aCL}$ positivity at high/medium titer. Nonetheless, $70 \%$ of aPL carriers described so far showed a concomitant autoimmune disease, being itself a risk factor for thrombotic events.

Objectives: We longitudinally followed up a cohort of healthy subjects persistently positive for aPL to evaluate the risk of developing thrombotic events.

Methods: Healthy subjects positive for aPL in at least 2 consecutive determinations ( $a P L$ carriers) were enrolled. Medical history was recorded and the following parameters were registered: presence of traditional risk factors (smoking, diabetes mellitus, hypertension, dyslipidemia, hormone therapy); obstetric history (infertility, miscarriages); family history of autoimmune and cardiovascular diseases with an early onset; recent infectious episodes. Laboratory evaluation was performed, including aCL $\operatorname{lgG} / \operatorname{lgM}$, anti- $\beta 2 \mathrm{GPI} \operatorname{lgG} / \operatorname{lgM}$, LA, antinuclear antibody, C3/C4 serum levels, thrombophilia screening. All subjects were evaluated every 6 months.

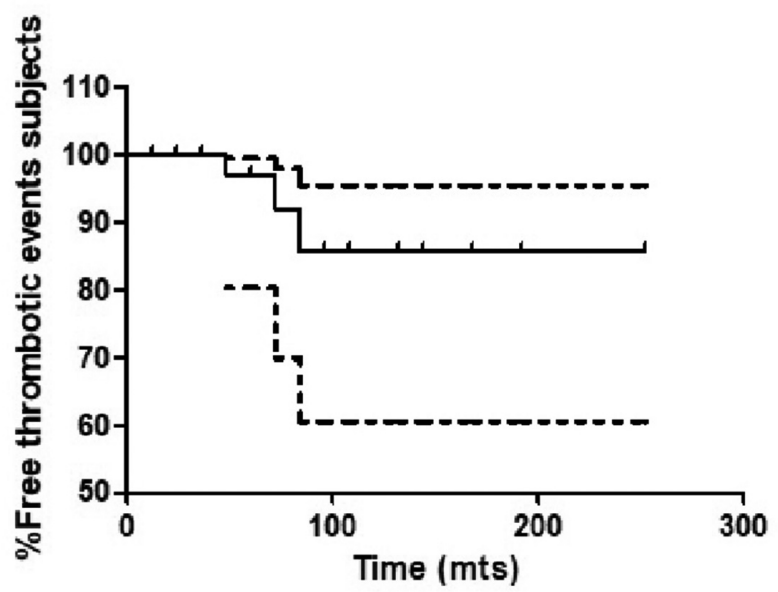

Abstract OP0121 - Figure 1. Reports the Kaplan-Meier curve of aPL carriers free from thrombotic events.

Results: We enrolled 47 aPL carriers (M/F 9/38, median age at first visit 45.5 years, IQR 17$)$. Thirty-six subjects $(76.6 \%)$ were $\mathrm{aCL}+(30.5 \%$ at medium/high titer), $30(63.8 \%)$ anti- $\beta 2 \mathrm{GPI}+(36.7 \%$ at medium/high titer), $24(51.1 \%) \mathrm{LA}+$. Thirty-one subjects $(65.9 \%)$ were positive for more than one aPL and $12(25.5 \%)$ showed a triple positivity. The aPL carriers were longitudinally followed up for a median of 60 months (IQR 48). Twenty-four subjects were treated with low dose aspirin (LDA) and 3 with hydroxychloroquine. During this observational period, 5 subjects were lost to follow-up and 5 became persistently negative for $\mathrm{APL}$ after a median of 54 months (IQR 39). Considering the remaining 37 aPL carriers, 3 (1F, $2 \mathrm{M}$ ) experienced a thrombotic event ( 2 arterial and 1 venous). This patients were treated with LDA at the time of the event and were all LA+. In one case, the episode was preceded by a flu-like event. Interestingly, the LA prevalence resulted significantly higher in the subjects experiencing thrombotic events in comparison with those who did not $(p=0.0001)$. We observed an absolute risk of thrombotic events of 0.08 ( $\mathrm{Cl}: 0.02$ to 0.2 ) and an incidence rate of 1.1 ( $\mathrm{Cl}: 0.3$ to 2.9$)$.

Conclusions: In this prospective study, specifically designed to evaluate the incidence of APS related clinical manifestations in aPL positive healthy subjects, LA positivity resulted the most important risk factor of thrombotic events.

Disclosure of Interest: None declared

DOI: 10.1136/annrheumdis-2018-eular.5829

\section{OP0122 \\ VALIDATION OF REMISSION AND LUPUS LOW DISEASE ACTIVITY STATE AS PREDICTORS OF ORGAN DAMAGE IN SLE}

M. Petri ${ }^{1}$, D.W. Goldman ${ }^{1}$, L.S. Magder ${ }^{2} .{ }^{1}$ Medicine, Johns Hopkins University School of Medicine; ${ }^{2}$ Biostatistics, University of Maryland, Baltimore, USA

Background: Outcome measures that combine control of SLE activity and prednisone reduction are clinically relevant. A clinical goal in SLE is to reduce risk of long-term organ damage.
Abstract OP0122 - Table 1. Rates of new damage, in subgroups defined by past levels of disease activity

\begin{tabular}{|c|c|c|c|c|c|}
\hline $\begin{array}{l}\text { Percentage } \\
\text { of Prior } \\
\text { Months in: }\end{array}$ & $\begin{array}{l}\text { Number of } \\
\text { person- } \\
\text { months } \\
\text { observed }\end{array}$ & $\begin{array}{c}\text { Number of months } \\
\text { with an increase in } \\
\text { SLICC/ACR Damage } \\
\text { Index }\end{array}$ & $\begin{array}{c}\text { Rate of } \\
\text { damage per } \\
100 \text { person } \\
\text { months }\end{array}$ & Rate Ratios & P-values \\
\hline Clinical & - & - & - & - & - \\
\hline Remission & 35772 & 406 & 1.13 & 1.0 (Ref) & - \\
\hline None & 14358 & 102 & 0.71 & 0.60 & $<0.0001$ \\
\hline Not none, & 6573 & 50 & 0.76 & $(0.48,0.75)$ & 0.023 \\
\hline but $<25 \%$ & 3845 & 27 & 0.70 & 0.66 & 0.035 \\
\hline $\begin{array}{l}25 \% \text { to } \\
50 \%\end{array}$ & 1641 & 10 & 0.61 & $\begin{array}{c}(0.46,0.94) \\
0.63\end{array}$ & 0.12 \\
\hline $50 \%$ to & & & & $(0.42,0.97)$ & \\
\hline $75 \%$ & & & & 0.58 & \\
\hline $75 \%+$ & & & & $(0.30,1.15)$ & \\
\hline Clinical & - & - & - & - & - \\
\hline Remission & - & - & - & - & - \\
\hline on & 16491 & 250 & 1.52 & 1.0 (Ref) & - \\
\hline Treatment & 20169 & 170 & 0.84 & 0.54 & $<0.0001$ \\
\hline None & 14344 & 103 & 0.72 & $(0.44,0.67)$ & $<0.0001$ \\
\hline Not none, & 8396 & 54 & 0.64 & 0.46 & $<0.0001$ \\
\hline but<25\% & 2789 & 18 & 0.65 & $(0.36,0.60)$ & 0.0019 \\
\hline $\begin{array}{l}25 \% \text { to } \\
50 \%\end{array}$ & & & & $\begin{array}{c}0.43 \\
(0.30,0.60)\end{array}$ & \\
\hline $50 \%$ to & & & & 0.45 & \\
\hline $75 \%$ & & & & $(0.27,0.75)$ & \\
\hline \multicolumn{6}{|l|}{$75 \%+$} \\
\hline LLDAS & - & - & - & - & - \\
\hline None & 30366 & 343 & 1.13 & 1.0 (Ref) & - \\
\hline Not none, & 10880 & 106 & 0.97 & 0.86 & 0.18 \\
\hline but<25\% & 5012 & 40 & 0.80 & $(0.69,1.07)$ & 0.037 \\
\hline $25 \%$ to & 8494 & 60 & 0.71 & 0.70 & 0.0010 \\
\hline $50 \%$ & 7527 & 46 & 0.61 & $(0.51,0.98)$ & $<0.0001$ \\
\hline $50 \%$ to & & & & 0.63 & \\
\hline $75 \%$ & & & & $(0.48,0.83)$ & \\
\hline \multirow[t]{2}{*}{$75 \%+$} & & & & 0.54 & \\
\hline & & & & $(0.40,0.73)$ & \\
\hline LLDAS on & - & - & - & - & - \\
\hline Treatment & 7656 & 117 & 1.53 & 1.0 (Ref) & - \\
\hline None & 10555 & 134 & 1.27 & 0.83 & 0.14 \\
\hline Not none, & 12686 & 129 & 1.02 & $(0.65,1.06)$ & 0.0013 \\
\hline but $<25 \%$ & 18151 & 133 & 0.73 & 0.66 & 0.0010 \\
\hline $25 \%$ to & 13141 & 82 & 0.62 & $(0.51,0.85)$ & $<0.0001$ \\
\hline $50 \%$ & & & & 0.48 & \\
\hline $50 \%$ to & & & & $(0.37,0.61)$ & \\
\hline $75 \%$ & & & & 0.40 & \\
\hline $75 \%+$ & & & & $(0.30,0.54)$ & \\
\hline
\end{tabular}

Objectives: We assessed whether two recently proposed disease activity outcomes were predictive of future damage.

Methods: For each month of follow-up in a large SLE cohort, we determined whether the patient was in Clinical Remission (as defined by the DORIS working group) or lupus low disease activity state (LLDAS) (as defined by Franklyn et al). Clinical Remission was defined as a PGA $<0.5$, clinical SLEDAI 0 and no prednisone or immunosuppressants. Clinical Remission on Treatment allowed for prednisone $\leq 5 \mathrm{mg} /$ day and immunosuppressant use. LLDAS was defined as a SLEDAI $\leq 4, P G A \leq 1.0$, no major organ activity, and no new activity. LLDAS on treatment allowed for prednisone use $\leq 7.5 \mathrm{mg} / \mathrm{d}$ and immunosuppressants. Damage was defined using the SLICC/ACR Damage index.

Results: There were 81118 person-months observed among 2026 patients (92\% female, 53\% Caucasian, 39\% African-American). table 1 shows the rates of damage, per person month, in subgroups defined by Remission or LLDAS.

Damage rates were relatively low when LLDAS was achieved at least $50 \%$ of the time. These rates were similar to those experienced by patients who met a more stringent treatment restriction with Remission on Treatment at least $50 \%$ of the time.

Conclusions: Percent time in LLDAS had a clear dose response for rate ratios of organ damage. The equivalence of LLDAS and DORIS remission on treatment is welcome news, as LLDAS on treatment $>50 \%$ of the time is an easier goal to achieve (3 times more person-months observed in our cohort) and more realistic as a clinical trial outcome.

Disclosure of Interest: None declared

DOI: 10.1136/annrheumdis-2018-eular.1988 